\title{
Determining workspace parameters for a new type of $6 R S S$ parallel manipulator based on structural and geometric models
}

\author{
Lucian Milica, Alexandru Năstase and Gabriel Andrei* \\ Dunărea de Jos University of Galaţi, Department of Mechanical Engineering, Domnească 111800201, \\ Galaţi, Romania
}

\begin{abstract}
Workspace geometric modelling of a new type of 6ㅅS parallel manipulator is described below. In the beginning, the researches undertaken in this area by other authors are highlighted and then a definition of this type of mechanisms is provided. The structural model of the 6RSS manipulator is briefly described. Inverse geometric model and translation subspace methods are used in order to determine the dimensions that define the workspace volume of the parallel manipulator. The reachable workspace is defined as a subset of the whole workspace in relation with the positions achieved by the characteristic point.
\end{abstract}

\section{Introduction}

In the last thirty years several studies on the applicability of parallel manipulators have been developed. Various applications of precision engineering such as lithography, microscopic assemblies, medical devices etc., require high-precision positioning in order to manipulate an object in different environments.

Regardless of the number of degrees of freedom, fewer [1-8] or more [9-12], the parallel mechanisms have proven their utility in various domains such as flight simulators, telescope position control, radar antennas, pointing devices [13].Parallel mechanisms have a robust construction and can move bodies of considerable mass and size at high speed, establishing a translational motion or rotation of the mobile platform. Compared to serial mechanisms, parallel manipulators have some special characteristics: high rigidity, high dynamic loading capacity, chain closed structure, high positional accuracy. Determining the workspace of a parallel manipulator is obviously an important issue in the design stage.

\section{Structural model of the mechanism $6 \underline{R S S}$}

A mobile structure of a parallel mechanism, as illustrated in Figure 1, consists of $n$ fixed curves $\left(c_{1}\right) \ldots\left(c_{n}\right), n$ spatial actuators, $b_{1} \ldots b_{\mathrm{n}}$, linked to a mobile platform by $n$ spherical joints, $B_{1} \ldots B_{\mathrm{n}}$. Links between $n$ spatial actuators $b_{1} \ldots b_{\mathrm{n}}$ and curves $\left(c_{1}\right) \ldots\left(c_{\mathrm{n}}\right)$ are achieved through $n$ "point-curve"-type joints denoted by $A_{1} \ldots A_{\mathrm{n}}$.

*Corresponding author: gabriel.andrei@ugal.ro 
According to the quantitative analysis of the structure mobility, the following parameters are defined:

- $m=n+1$ ( $m$ is the number of mobile kinematic elements);

- $k_{2}=n$ (number of $A_{\mathrm{j}}$ kinematic joints of second class);

- $k_{3}=n$ (number of the $B_{\mathrm{j}}$ kinematic joints of third class).

The degree of mobility of the mechanism is given by Grübler-Kutzbach formula:

$$
M=6 \cdot m-\sum_{n=1}^{5} c \cdot k_{n}
$$

where:

- $c$ is the kinematic class of joints;

- $k_{\mathrm{n}}$ is the number of joints of $n^{\text {th }}$ class.

In the mechanical structure shown in Figure 1, the degree of mobility is:

$$
M=6 \cdot m-\sum_{n=1}^{5} c \cdot k_{n}=6(n+1)-2 n-3 n=n+6
$$

Present approach deals with the workspace of the mobile platform of a $\underline{R} S S$ manipulator with six degrees of freedom in different situations, when the constraints imposed on the platform are fully determined, limited or non-existent.

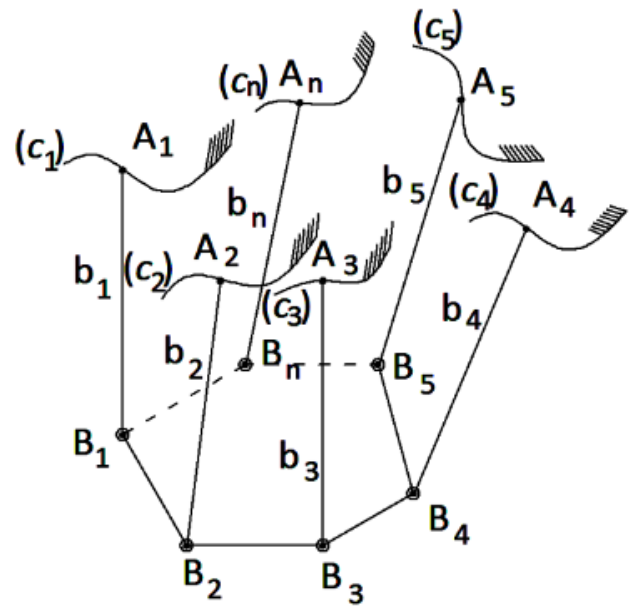

Fig. 1. Generic structural scheme of the parallel mechanism.

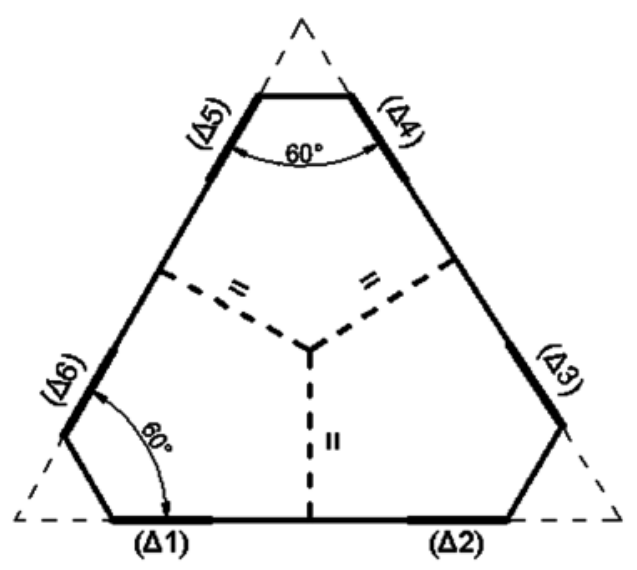

Fig. 2. Positioning of the axes of the rotational joints.

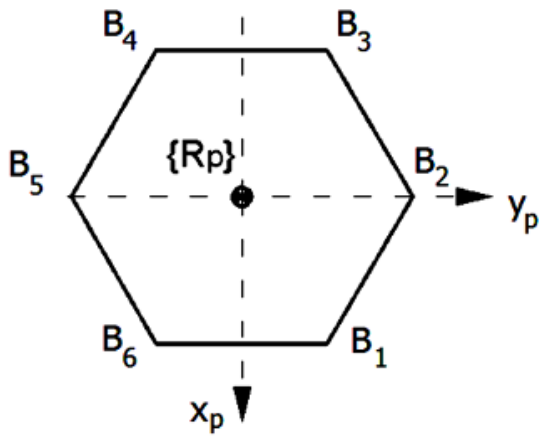

Fig. 3. Positioning of the spherical joints of the mobile platform. 


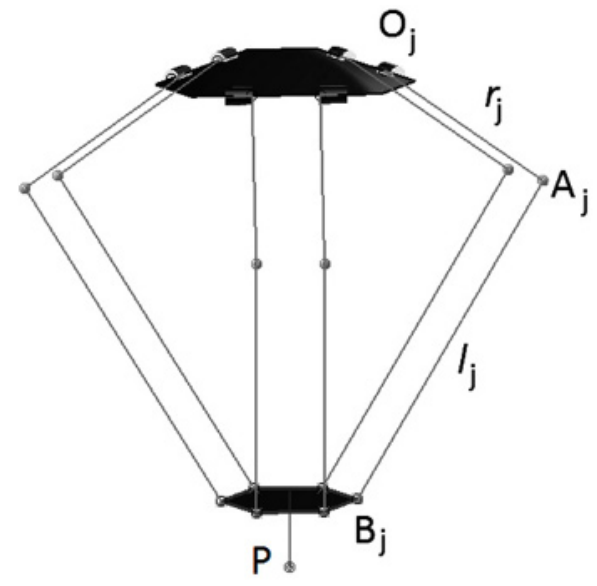

Fig. 4. Mechanism kinematic scheme.

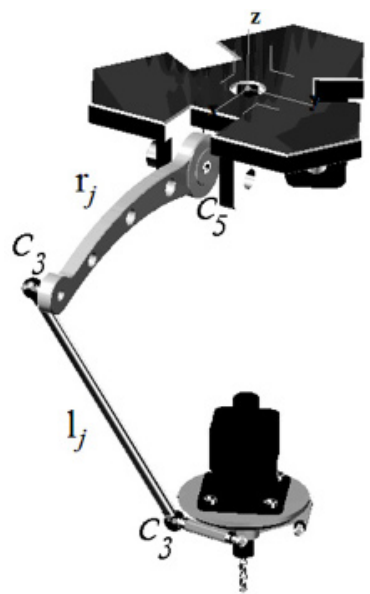

Fig. 5. New design version of $6 \underline{R S S}$ parallel manipulator (only a single actuating branch viewed).

The mechanism is characterized by the following features:

- the axis of rotational active joints $\Delta_{\mathrm{j}}$ that generates circular trajectories $\left(c_{\mathrm{j}}\right)$ are coplanar, twos coincident and disposed central-symmetrically (Fig. 2);

- the spherical joints of the mobile platform are coplanar and their centres are located on the corners of a regular hexagon (Fig. 3);

- the actuating arms $r_{\mathrm{j}}$ are of equal length (Fig. 4);

- the rods $l_{\mathrm{j}}$ are of equal length (Fig. 4);

- the distance between the spherical joints $A_{\mathrm{j}}$ and $A_{\mathrm{j}+1}$ is equal to the distance between $B_{\mathrm{j}}$ and $B_{\mathrm{j}+1}, j=1,3,5$ (Fig. 4).

A new design version of $6 \underline{R S S}$ parallel manipulator consists of a fixed plate with six servomotors, and a mobile platform on which a seventh servomotor is fitted to drive a milling tool (Fig.5).

\section{Inverse geometric model}

In the case of inverse geometric model, parameters $x, y, z, \alpha, \beta, \gamma$ of $\mathbf{T}_{\mathrm{P}, \mathrm{B}}$ matrix - giving the mobile platform position - are known while parameters $\theta_{1}{ }^{\mathrm{k}} \ldots \theta_{6} \mathrm{k}$ of each kinematic chain are to be determined.

In the following section, an algorithm for determining the angular parameter $\delta$ for one actuator will be described. A Cartesian coordinate system of versors $(\mathbf{v}, \mathbf{u}, \mathbf{k})$ is attached to cylindrical joint $C$, where $\mathbf{u}$ is the unit vector of the joint axis, $\mathbf{k}$ is parallel to $z_{0}$ fixed axis of the reference system $\left(x_{0}, y_{0}, z_{0}\right)$, and $\mathbf{v}=\mathbf{u} \times \mathbf{k}$.

Furthermore, from Figure 6 it can be noticed the projection $B^{\prime}$ of point $B$ on the plane determined by the unit vectors $\mathbf{v}$ and $\mathbf{k}$ that contains point $A$.

The algorithm of inverse geometrical model includes the following steps (Fig. 7):

1. Calculating the coordinates of point $B$ using the relation:

$$
\mathbf{r}_{\mathrm{B}}=\mathbf{T}_{\mathrm{P}}^{0} \cdot \mathbf{b}
$$

where $\mathbf{b}$ is the vector of local coordinates of the point $B$ in the platform reference frame.

2. Determining the distance $d$ between point $B$ and plan $[\mathbf{k ,}, \mathbf{v}]$ :

$$
d=\left(\mathbf{r}_{\mathrm{B}}-\mathbf{r}_{\mathrm{C}}\right) \cdot \mathbf{u}
$$




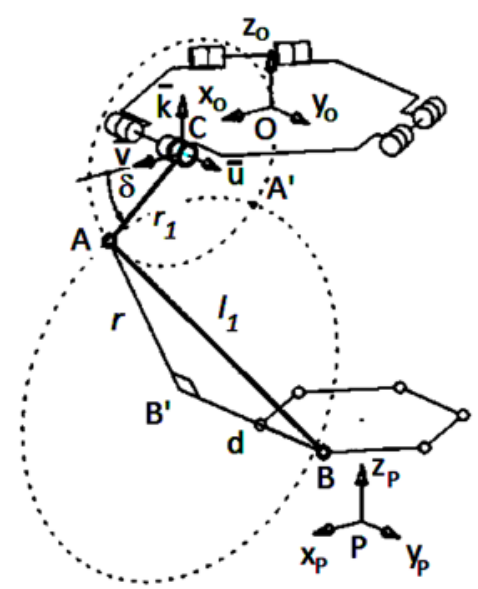

Fig. 6. Sketch of a single kinematic chain of the parallel manipulator 6 RSS.

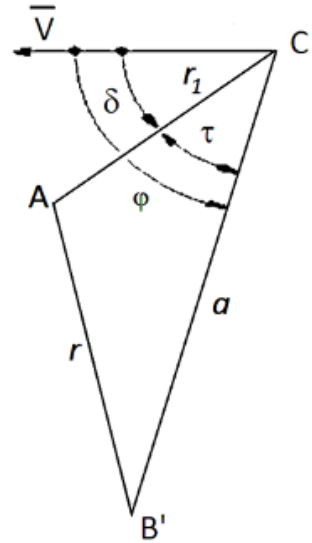

Fig. 7. Highlighting the angles $\varphi, \delta$ and $\tau$.

If $|d| \geq l_{1}$, jump out to point 6 .

3. Calculating the coordinates of point B':

$$
\mathbf{r}_{\mathrm{B} \prime}=\mathbf{r}_{\mathrm{B}}-d \cdot \mathbf{u}
$$

4. Calculating the distances $\mathrm{a}=\mathrm{B}^{\prime} \mathrm{C}$ and $\mathrm{r}=\mathrm{B}^{\prime} \mathrm{A}$ :

$$
a=\left|\mathbf{r}_{\mathrm{C}}-\mathbf{r}_{\mathrm{B}}\right| \text { and } r=\left|\mathbf{r}_{\mathrm{A}}-\mathbf{r}_{\mathrm{B}}\right|
$$

If $\mathrm{a} \geq r+r_{1}$ or $\mathrm{a} \leq r-r_{1}$, jump out to point 6 .

5. Calculating the value of angle $\varphi$.

Resolving $A B^{\prime} C$ triangle, and determining angle $\tau$. Obtaining the solution $\delta=\varphi-\tau$. End of algorithm.

6. There is no solution. End of algorithm.

\section{Translation subspace}

In a particular case when the mobile platform is parallel to the fixed platform and the axes of their associated coordinate systems are parallel to each other, then adjacent actuating arms are parallel $\left(\theta_{1}=\theta_{2}, \theta_{3}=\theta_{4}\right.$ and $\left.\theta_{5}=\theta_{6}\right)$, at any time of movement. Based on the above observation, a method for determining the working space through a geometric demonstration about the locus of $B_{\mathrm{k}}$ points has been developed (Fig. 8). The method presented below is available only under specified conditions, applied just for a single linkage chain (e.g. $O_{\mathrm{k}} A_{\mathrm{k}} B_{\mathrm{k}}$ ). The restriction imposed by the stiffness of the actuating arm $A_{\mathrm{k}} B_{\mathrm{k}}$, i.e. constant distance between points $A_{\mathrm{k}}$ and $B_{\mathrm{k}}$, can be interpreted as a locus of $A_{\mathrm{k}}$ positions in relation to $B_{\mathrm{k}}$, which is a spherical surface (Fig. 8a).

Then the locus of point $B_{\mathrm{k}}$ will be the volume generated by the sphere defined above, when it sweeps the arc of radius $r_{\mathrm{k}}=O_{\mathrm{k}} A_{\mathrm{k}}$ and angle $\theta_{\mathrm{h}}$ (Fig. 8b). Figure 9 shows a section through a plane normal to the axis of rotation of the arm. $A_{0}$ and $A_{1}$ are the centre of the sphere at initial and end position, respectively. Spherical surface was divided into two semispheres, $S_{1}$ and $S_{2}$. Figure 9a presents the section of the volume resulted after the displacement of the spherical surface. It is noticed that in this figure there is an empty space inside. The volumes generated by $S_{1}$ and $S_{2}$, whose intersection is the volume investigated, can be seen in Figure 9b. The reachable workspace is defined by the totality of space positions, orientations and locations that can be achieved by the characteristic point in a certain configuration of the actuating arms. This space is a subset of the whole workspace. 


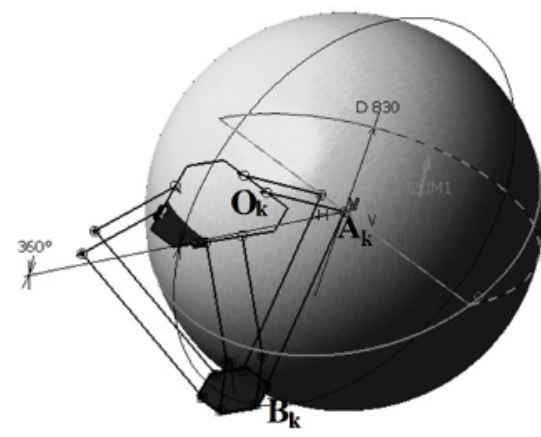

a

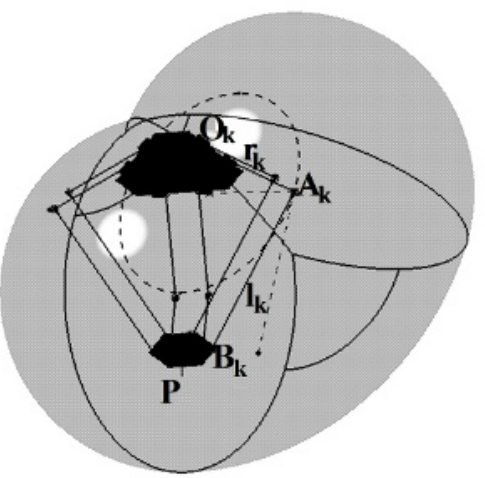

b

Fig. 8. Spherical surface generated by locus of point $B_{\mathrm{k}}$ (a); the volume swept when the sphere is moving on the arc with radius $r_{\mathrm{k}}(\mathrm{b})$.

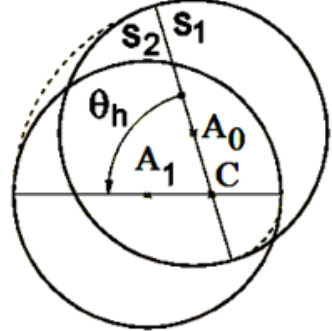

a

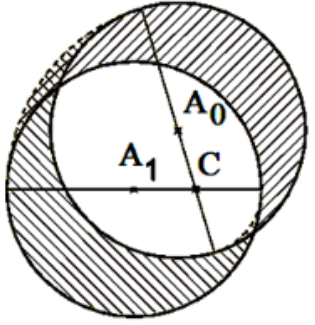

b

Fig. 9. Sections through the locus of point $B_{\mathrm{k}}$ : (a) the section of the volume resulted; (b) projection of the intersection of volumes.

Actual determination of reachable workspace was done with a software application written in AutoLISP. Using the same AutoLISP code, it is possible to determine the reachable workspace of the parallel mechanism 6RSS, for different combinations of parameters $a_{1}, b_{1}, a_{2}, b_{2}, r_{1}, l_{1}, h, \theta_{\mathrm{oc}}$. Furthermore, a simulating approach is possible when certain positions must be reached during a particular manufacturing operation.

\section{The volume of the translational workspace}

In order to maximize the workspace volume, an algorithm for determining the boundaries of the operating space has been developed. The proposed algorithm consists of the following steps:

- cutting the reachable workspace with an horizontal plane of an arbitrary elevation, " $z$ ";

- in the section obtained, determining the vertices of an inscribed rectangle of maximum area, $x_{\mathrm{opt}} \times y_{\mathrm{opt}}$; the product between elevation $" z$ " and rectangle area is the volume of a parallelepiped $V_{\text {opt, }}$ which is maximum, related to the section plane (Fig.10);

- repeating previous steps for a number of " $z$ " values and determining a volume chart.

The maximum-maximorum value of the volume chart will be the searched volume with entailed boundaries.

Further, an AutoLISP program to perform the above algorithm for a large number of equidistant plans was written (Fig.11). Using the program, an analysis for 20 elevation plans was carried out. Then, the chart of the maximum inscribed volume, depending on the elevation " $z$ " of the current plan, was accomplished. The graph in Figure 12 shows the value of maximum-maximorum volume. 


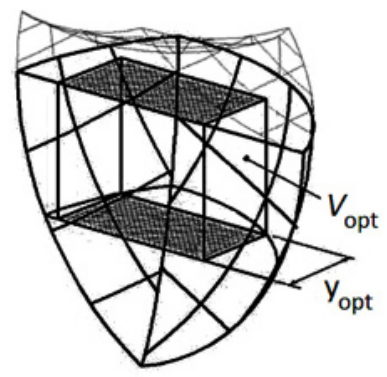

Fig. 10. Determining the rectangle of maximum area.

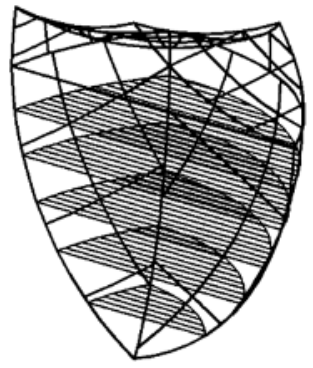

Fig.11. Equidistant sectioning of the reachable workspace.

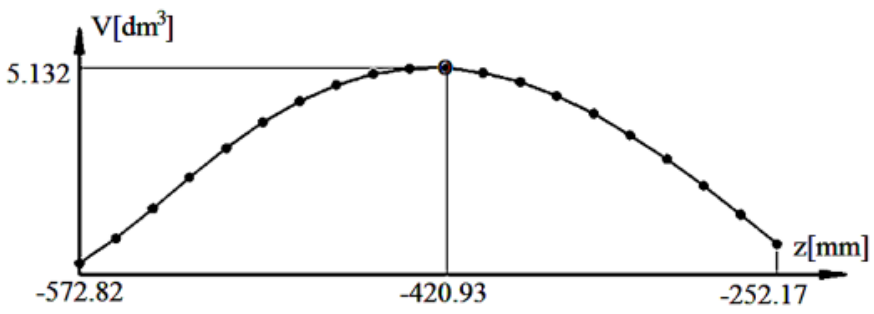

Fig. 12. Volume chart and the maximum-maximorum value.

\section{Conclusions}

The inverse geometric model and the subspace of translations were used in order to develop algorithms and software applications for determining optimal workspace of $6 \underline{\mathrm{R} S S}$ parallel manipulator. The method described above is useful to assess the performance of parallel manipulators designed according to a certain structure and it can be extrapolated for other structural schemes. Using such algorithms, extreme values of optimal translational workspace may be inferred. Therefore a better understanding of the operation capability of parallel mechanisms could be achieved. Accurately defined dimensions for the translational workspace entails the range of kinematics parameters.

\section{References}

1. B. Roger, N. Scott, Mech. Mach. Theory, 56, 138-155. (2012)

2. Y. Zhang, Q.Liao, H. Su,S.Wei, Mech. Mach. Theory, 52, 47-58 (2012)

3. C. Lee, M. Jacques, Mech. Mach. Theory,51, 185-194 ( 2012)

4. R. Amir, A. Alireza, R. Mohammad, Mech. Mach. Theory, 51, 195-216(2012)

5. S. Liu, Y. Yu, Z. Zhu, L. Su, Q. Liu, J. Cent. South. Univ. T., 17, 323-331 (2010)

6. Q. Li., Q. Chen,C. Wu,Z. Huang, Mech. Mach. Theory, 65, 46-57 (2013)

7. R. Srivatsan, B. Sandipan, Mech. Mach. Theory,62, 150-165 (2013)

8. Z. Du, R. Shi, W. Doung, J. Cent. South. Univ. T, 19, 2482-2487 (2012)

9. H.S. Kim, L.W. Tsai, ASME J. Mech.Des. 125, 92-97 (2003)

10. C.H. Liu, S. Cheng, ASME J. Mech. Des. 126,1006-1016 (2004)

11. I.A. Bonev, J. Ryu,Mech. Mach. Theory, 36, 1-13 (2001)

12. L. Milica, G. Andrei, MATEC web of conf.,94(2017)

13. D. Zhang, Parallel robotic machine tools, Springer(2010) 